\title{
Electrically pumped edge-emitting photonic crystal lasers with angled facets
}

\author{
Lin Zhu, Guy A. DeRose, Axel Scherer, and Amnon Yariv \\ Department of Electrical Engineering, California Institute of Technology, 1200 E. California Boulevard, \\ Pasadena, California 91125, USA
}

Received January 17, 2007; accepted February 1, 2007;

posted February 16, 2007 (Doc. ID 79091); published April 17, 2007

\begin{abstract}
We demonstrate electrically pumped large-area edge-emitting InGaAsP/InP two-dimensional photonic crystal lasers with angled facets at room temperature. The laser uses a weak index perturbation surface photonic crystal structure to control optical modes in the wafer plane. Measurements of the laser spectra show that the modal selection is due to satisfying the Bragg resonance conditions in both the longitudinal and the transverse directions. The lasing wavelength is tuned lithographically by changing photonic crystal lattice constants. We demonstrate a fine lasing wavelength tuning sensitivity (change of lasing wavelength over change of lattice constant) of 0.08 through the transverse lattice constant tuning. () 2007 Optical Society of America

OCIS codes: $140.5960,350.2770,130.2790$
\end{abstract}

It is well known that photonic crystal waveguiding structures can support two different kinds of modes: effective index guided and Bragg guided. For effective index-guided modes, in photonic crystal fibers, for example, ${ }^{1}$ confinement in the transverse direction is due to the index difference between the core and the effective medium formed by the periodic structure in the cladding. We have recently demonstrated single-mode lasing based on effective index guiding for on-chip photonic crystal lasers. ${ }^{2}$ For Bragg-guided modes, light is confined by Bragg reflection arising from a photonic bandgap in the transverse direction. Lasing with Bragg-guided modes can be designed to maintain a near-diffraction-limited far field for largearea edge-emitting semiconductor lasers even at relatively high powers. ${ }^{3-7}$ For these lasers, angled facets are proposed to suppress effective indexguided modes and gain-guided modes and to ensure the lasing of Bragg-guided modes only. ${ }^{3-5}$ Optically pumped mid-infrared semiconductor photonic crystal lasers $(\lambda \sim 3.7 \mu \mathrm{m})$ with Bragg-guided modes have been demonstrated at low temperatures. ${ }^{8}$

In this Letter, we describe electrically pumped large-area edge-emitting photonic crystal lasers with angled facets in the InGaAsP/InP semiconductor material at room temperature. We use a small index contrast for the photonic crystal structure to allow a weak Bragg confinement and thus a large-area operation. We measure laser spectra and far fields with pulsed current injection. We lithographically tune the lasing wavelength by changing the lattice constants in both the longitudinal and the transverse directions. The experimental results agree well with the theoretical calculations for the lasing wavelength. We also show that the transverse lattice constant tuning can provide a tuning sensitivity (change of lasing wavelength over change of lattice constant) of 0.08 , which is $\sim 30$ times smaller than that of a regular distributed feedback (DFB) laser or a photonic crystal laser with large index contrasts. 9,10

Figure 1(a) shows a schematic of our photonic crystal laser. The photonic crystal consists of a rectangular lattice array of polymer-filled holes each with a radius of $100 \mathrm{~nm}$ on the wafer surface. The transverse and longitudinal lattice constants are $a$ and $b$, respectively. The cleaved facet is titled relative to the transverse lattice direction with an angle $\theta_{\text {tilt. }}$. In the wafer plane $(x-z)$, the transverse $(x)$ Bragg resonance condition defines the guiding mechanism while the longitudinal $(z)$ Bragg resonance condition selects a particular longitudinal mode. ${ }^{7,11}$ This Bragg condition can be expressed as: $k_{x}=l \pi / a, k_{z}=j \pi / b$, where $k_{x}$ is the transverse wave vector, $k_{z}$ is the longitudinal wave vector, and $l, j$ are nonzero integers. In the limit of weak index perturbation, we can use the effective index method to simplify our analysis. Thus $k_{x}$ and $k_{z}$ satisfy

$$
\begin{gathered}
k_{x}^{2}+k_{z}^{2}=n_{\mathrm{eff}}^{2} k_{0}^{2}=n_{\mathrm{eff}}^{2}(2 \pi / \lambda)^{2}, \\
\lambda=2 n_{\mathrm{eff}}\left(l^{2} / a^{2}+j^{2} / b^{2}\right)^{-1 / 2},
\end{gathered}
$$

where $n_{\text {eff }}$ is the effective refractive index for the optical mode of the wafer epitaxial structure and $\lambda$ is the resonance (lasing) wavelength. We can define the transverse plane resonance angle $\theta_{\text {res }}$ as: $\theta_{\text {res }}(\lambda)$ $=\cos ^{-1}\left[\sqrt{\left(2 \pi n_{\text {eff }} / \lambda\right)^{2}-(l \pi / a)^{2}} /\left(2 \pi n_{\text {eff }} / \lambda\right)\right]$ and the facet tilt angle $\theta_{\text {tilt }}$ is chosen to be same as the resonance angle $\theta_{\text {res }}$ at $1550 \mathrm{~nm}^{11}$

The fabrication procedure consists of two main steps: the definition of large-area photonic crystal patterns and the polyimide planarization. ${ }^{2}$ The photonic crystal structure is first written on the wafer surface by electron-beam lithography and is then etched into the semiconductor for $\sim 360 \mathrm{~nm}$ to obtain the desired index contrast. The polyimide planarization is realized by an etch-back process and creates a polyimide post inside each etched hole. The polyimide separates the etched holes from the subsequently evaporated metal contact to reduce the optical loss and help obtain good contact quality. Figure 1(b) shows a scanning electron microscope image of a photonic crystal laser after the polyimide planarization.

We first use a first-order Bragg reflection $(l=1)$ for the transverse direction with a lattice constant of $a$ $=1 \mu \mathrm{m}$ and a second-order Bragg reflection $(j=2)$ for 
the longitudinal direction with three different lattice constants of $b=480,490$, and $500 \mathrm{~nm}$. The metal contact width is $\sim 100 \mu \mathrm{m}$. The tilt angle is $13.8^{\circ}$ for all three designs. The laser bars are cleaved to lengths of $\sim 480 \mu \mathrm{m}$ and are tested in pulsed operation at room temperature with no active cooling. Current pulses with a duration of $100 \mathrm{~ns}$ and a period of $10 \mu \mathrm{s}$ are injected to drive the lasers.

Lasing is obtained for the $b=490$ and $500 \mathrm{~nm}$ designs with different threshold current densities of $J$ th $=1.10$ and $1.33 \mathrm{kA} / \mathrm{cm}^{2}$, respectively. Figure $2(\mathrm{a})$ shows the optical spectra for all three designs at the same pump current density $J=1.40 \mathrm{kA} / \mathrm{cm}^{2}$. While lasing is not obtained for the device with $b=480 \mathrm{~nm}$, the resonance peak at $1523.1 \mathrm{~nm}$ is evident in the spectrum. The $b=480 \mathrm{~nm}$ design does not lase because the resonance peak wavelength for this design is far away from the peak wavelength of the gain

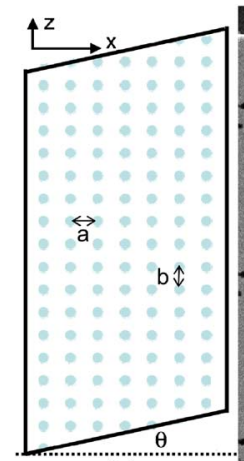

(a)

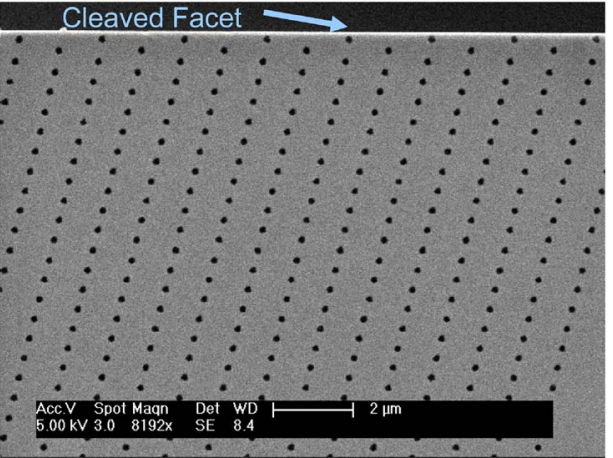

(b)
Fig. 1. (Color online) (a) Schematic of a two-dimensional photonic crystal laser. $a$ is the transverse lattice constant, $b$ is the longitudinal lattice constant, and $\theta_{\text {tilt }}$ is the facet tilt angle. (b) SEM image of the photonic crystal laser with a cleaved facet. Each etched hole is filled with a planarization polymer. The image is taken before the deposition of electrical contacts.

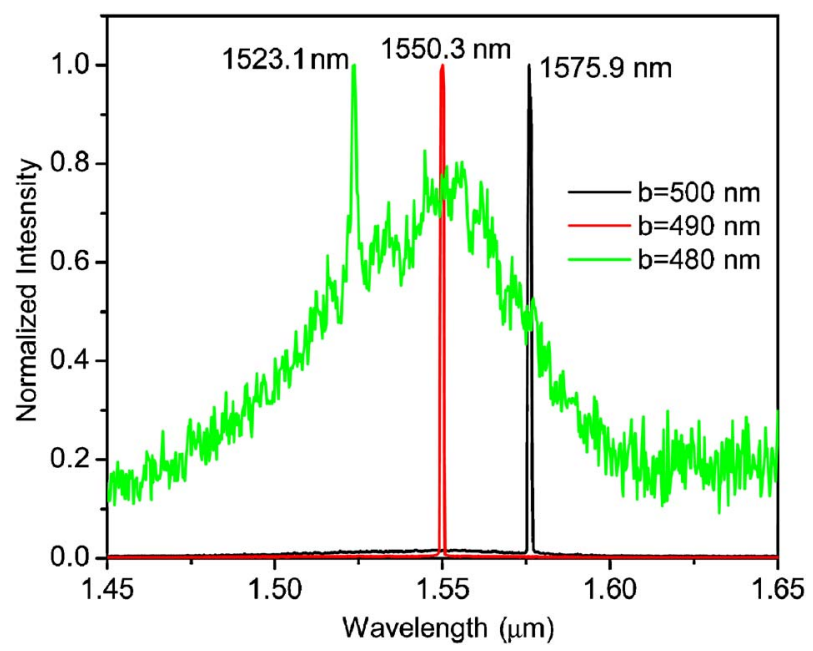

Fig. 2. (Color online) Optical spectra for the lasers with the same transverse lattice constant but different longitudinal lattice constants at $J=1.40 \mathrm{kA} / \mathrm{cm}^{2}$. Lasing is obtained for the 490 and $500 \mathrm{~nm}$ designs with lasing wavelengths of 1550.3 and $1575.9 \mathrm{~nm}$, respectively. The resonance peak at $1523.1 \mathrm{~nm}$ for the $480 \mathrm{~nm}$ design is evident in the spectrum, although lasing is not obtained. spectrum and intrinsic losses at short wavelengths are high.

In all three designs, the experimental resonance wavelengths of $1523.1,1550.3$, and $1575.9 \mathrm{~nm}$ are close to the theoretical predictions of 1520.2, 1550.1, and $1579.9 \mathrm{~nm}$ calculated from Eq. (1). $n_{\text {eff }}$ is chosen to be 3.257 in the calculation, and it is numerically calculated by a mode solver for the wavelength of $1550 \mathrm{~nm}$. The difference at 1523.1 and $1575.9 \mathrm{~nm}$ is mainly due to the material dispersion. Tuning of the longitudinal lattice constant leads to a lasing wavelength tuning sensitivity of 2.6 , which is similar to that of regular second-order DFB lasers and photonic crystal lasers with large index contrasts.

Figure 3(a) shows the spectra for the $b=490 \mathrm{~nm}$ design with different pumping currents. As the pumping current increases, the lasing spectrum shifts to longer wavelengths and the linewidth increases due to excessive heating. In spite of the smooth singlepeaked spectra in Fig. 3(a), we cannot authoritatively draw conclusions about single or multimodedness. Due to the pulsed excitation, the spectra are obtained under transient conditions with the attendant broadening. Also the resolution of our optical spectrum analyzer $(\sim 0.1 \mathrm{~nm})$ is marginal for the task. The farfield profile at the $3.5 \times$ threshold shown in Fig. 3(b) reveals possible non-single-mode operation. It has a $1.7 \times$ diffraction-limited (we assume the emitting aperture is $100 \mathrm{~mm}$ ) divergence angle of $1.8^{\circ}$ and two parasitic side peaks. Three main reasons account for the possible non-single-mode behavior: pulsed pump currents ${ }^{8}$ current leakage outside the contact region, $, 4,12$ and unwanted Bragg reflection due to fabrication errors.

Transverse and longitudinal Bragg conditions need to be satisfied simultaneously for our lasers. Thus we can also tune the lasing wavelength by changing the transverse lattice constant. We can calculate the tuning sensitivity for both the longitudinal and the transverse directions from Eq. (1):

$$
\begin{aligned}
& \mathrm{d} \lambda / \mathrm{d} a=2 n_{\text {eff }}\left(l^{2} / a^{2}+j^{2} / b^{2}\right)^{-3 / 2}\left(l^{2} / a^{3}\right), \\
& \mathrm{d} \lambda / \mathrm{d} b=2 n_{\text {eff }}\left(l^{2} / a^{2}+j^{2} / b^{2}\right)^{-3 / 2}\left(j^{2} / b^{3}\right) .
\end{aligned}
$$

In our designs, the transverse wave vector is much smaller than the longitudinal wave vector. This means that we can finely tune the lasing wavelength with a relatively large step of the transverse lattice constant. To show this advantage, we fabricate lasers with the same longitudinal constant of $b=490 \mathrm{~nm}$ and five different transverse lattice constants of $a$ $=0.92,0.96,1.0,1.04$, and $1.08 \mu \mathrm{m}$ on the same chip. Other fabrication parameters are the same as the previous design. Figure 4(a) shows the lasing spectra at $\sim 1.5 \times$ threshold for all five lasers. We also show the lasing wavelength as a function of the transverse lattice constant and the corresponding theoretical calculations in Fig. 4(b). As the transverse lattice constant changes from 0.92 to $1.08 \mu \mathrm{m}$, the lasing wavelength shifts $12.7 \mathrm{~nm}$. This corresponds to a transverse tuning sensitivity of $0.08,30$ times smaller than the longitudinal tuning sensitivity. The 

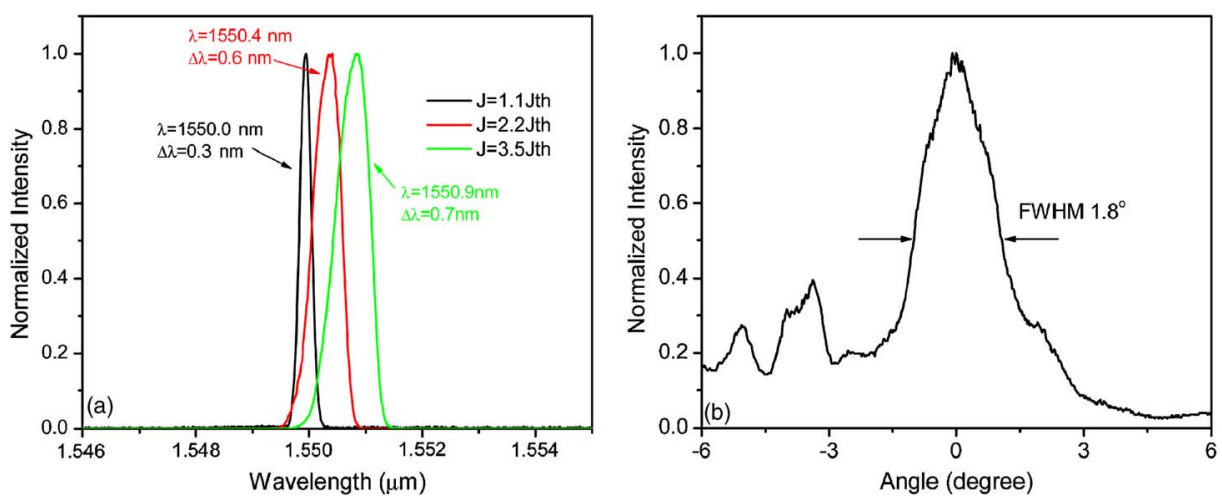

Fig. 3. (Color online) (a) Lasing spectra for the $490 \mathrm{~nm}$ design at different pump currents $(1.1 \times, 2.2 \times$, and $3.5 \times$ threshold). Further increase of the pump current is limited by our current source. (b) Corresponding far-field profile for the $490 \mathrm{~nm}$ design at the $3.5 \times$ threshold.
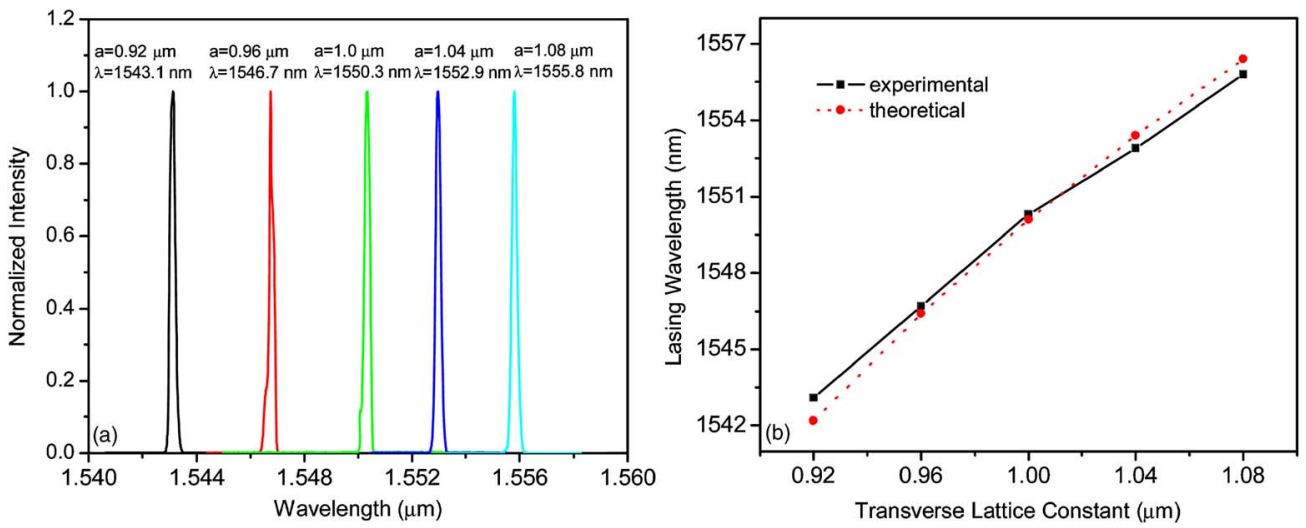

Fig. 4. (Color online) (a) Lasing spectra for the lasers with the same longitudinal lattice constant but different transverse lattice constants at the $\sim 1.5 \times$ threshold. A small tuning sensitivity of 0.08 is obtained. (b) Comparison between the experimental and theoretical results for the lasing wavelength. $n_{\text {eff }}$ is also chosen to be 3.257 in the calculation. A good match is obtained, and the slight difference is mainly due to the material dispersion.

small tuning sensitivity is important for accurate control of the lasing wavelength.

In summary, we have demonstrated electrically pumped large-area edge-emitting photonic crystal lasers in InGaAsP active semiconductor materials at room temperature. Lasing with a narrow spectrum linewidth of $0.7 \mathrm{~nm}$ and a $1.7 \times$ diffraction-limited far-field divergence angle of $1.8^{\circ}$ is obtained at a $3.5 \times$ threshold for $100 \mu \mathrm{m}$ wide and $480 \mu \mathrm{m}$ long devices. We finely tune the lasing wavelength by changing the transverse lattice constant and achieve a tuning sensitivity of $0.08,30$ times smaller than that of a regular DFB laser or a photonic crystal laser with large index contrasts.

L. Zhu thanks O. Painter and R. Perahia for providing access to their plasma-enhanced chemical vapor deposition facility. P. Chak, J. Choi, and J. Poon are acknowledged for fruitful discussions. The work was supported by the Air Force Office of Scientific Research (H. Schlossberg) and by the Defense Advanced Research Projects Agency (J. Mangano). L. Zhu's e-mail address is linz@caltech.edu.

\section{References}

1. T. A. Birks, J. C. Knight, and P. St. J. Russell, Opt. Lett. 22, 961 (1997).
2. L. Zhu, P. Chak, J. K. S. Poon, G. A. DeRose, A. Yariv, and A. Scherer (submitted to Opt. Express).

3. R. J. Lang, K. D. Zurko, A. Hardy, S. Demars, A. Schoenfelder, and D. Welch, IEEE J. Quantum Electron. 34, 2196 (1998).

4. A. M. Sarangan, M. W. Wright, J. Marciante, and D. J. Bossert, IEEE J. Quantum Electron. 35, 1220 (1999).

5. I. Vurgaftman and J. R. Meyer, IEEE J. Quantum Electron. 38, 592 (2002).

6. A. Yariv, Opt. Lett. 27, 936 (2002).

7. L. Zhu, J. M. Choi, G. A. DeRose, A. Yariv, and A. Scherer, Opt. Lett. 31, 1863 (2006).

8. C. S. Kim, W. W. Bewley, C. L. Canedy, I. Vurgaftman, M. Kim, and J. R. Meyer, IEEE Photon. Technol. Lett. 16, 1250 (2004).

9. M. Nakamura, H. W. Yen, A. Yariv, E. Garmire, S. Somekh, and H. L. Garvin, Appl. Phys. Lett. 23, 224 (1973).

10. O. Painter, A. Husain, A. Scherer, P. T. Lee, I. Kim, J. D. O’Brien, and P. D. Dapkus, IEEE Photon. Technol. Lett. 12, 1126 (2000).

11. L. Zhu, A. Yariv, and A. Scherer (submitted to IEEE J. Quantum Electron).

12. K. Paschke, A. Bogatov, F. Bugge, A. E. Drakin, J. Fricke, R. Güther, A. A. Stratonnikov, H. Wenzel, G. Erbert, and G. Tränkle, IEEE J. Sel. Top. Quantum Electron. 9, 1172 (2003). 